\title{
Degradation of Functional Materials in Temperature Gradients - Thermodiffusion and the Soret Effect
}

\author{
Jürgen Janek ${ }^{\dagger}$, Joachim Sann, Boris Mogwitz, Marcus Rohnke, and Matthias Kleine-Boymann \\ Physikalisch-Chemisches Institut, Justus Liebig University Gießen, 35392 Gießen, Germany \\ (Received January 3, 2012; Accepted February 2, 2012)
}

\begin{abstract}
Functional materials are often exposed to high temperatures and inherent temperature gradients. These temperature gradients act as thermodynamic driving forces for the diffusion of mobile components. The detailed consequences of thermodiffusion depend on the boundary conditions of the non-isothermal sample: Once the boundaries of the sample are inert and closed for exchange of the mobile components, thermodiffusion leads to their pile-up in the stationary state (the so called Soret effect). Once the system is open for an exchange of the mobile component, chemical diffusion adds to the Soret effect, and stationary non-zero component fluxes are additionally observed in the stationary state. In this review, the essential aspects of thermodiffusion and Soret effect in inorganic functional materials are briefly summarized and our current practical knowledge is reviewed. Major examples include nonstoichiometric binary compounds (oxides and other chalcogenides) and ternary solid solutions. The potential influence of the Soret effect on the long term stability of high temperature thermoelectrics is briefly discussed. Typical Soret coefficients for nonstoichiometric compounds are found to be of the order of $(\mathrm{d} \delta / \mathrm{dT}) \approx 1 \% / \mathrm{K}$.
\end{abstract}

Key words : Soret effect, Thermodiffusion, Thermoelectrics, Degradation

\section{Introduction}

I $\mathrm{t}$ is well known that a temperature gradient not only induces heat transport but also induces the transport of matter (thermodiffusion, Ludwig-Soret effect) in systems containing mobile components (see Allnatt and Chadwick, ${ }^{1)}$ Jost $^{2)}$ and Allnatt and Lidiard ${ }^{3)}$ for some historical remarks on solid state thermodiffusion). Thus, originally homogeneous mixtures demix in a temperature gradient. This effect is used quite effectively for the separation of gaseous isotopes in the so-called Clausius separation tube, is of interest in the field of fluids and polymers, but has also great practical relevance in the field of high temperature materials and thermoelectrics exposed to steep temperature gradients.

Surprisingly, our empirical knowledge on thermodiffusion in functional materials is scarce, despite a long history of experimental studies. Systematic research in thermodiffusion and the related ionic thermopower in the solid state started with the experiments of Reinhold et al. on the demixing of silver and copper halides and chalcogenides in a temperature gradient $\left(\right.$ Reinhold $\left.{ }^{4}\right)$. It was the time of Wagner and Schottky who established the field of defect thermodynamics, and a number of fundamental studies of defect-related crystal properties were performed. ${ }^{5)}$ However, due to ill-defined experimental conditions (mostly the applied temperature range

\footnotetext{
${ }^{\dagger}$ Corresponding author : Jürgen Janek

E-mail : juergen.janek@phys.chemie.uni-giessen.de

Tel : +82-49-641-99-34501 Fax : +82-49-641-34509
}

included a miscibility gap), no reliable results on the Soret effect were obtained. Later, following new theoretical work by Howard and Lidiard, ${ }^{6}$ the measurement of thermopower became a standard tool in this study of point defects. Major contributions were reported by Christy, ${ }^{7)} \mathrm{Haga}^{8)}$ and Wagner. ${ }^{9)}$

Extensive work on thermomigration in metals was stimulated by experimental observations during electromigration studies in the 1960's. The need for high current densities in the electromigration experiments led to large temperature gradients and a superposition of both effects. A survey of the early studies in this field was given by Wever in $1973 .{ }^{10)}$ More recent studies and surveys of the field were reported by Bocquet et al. ${ }^{11)}$ and Faupel. ${ }^{12)}$

New interest in thermomigration in ionic compounds in the 1960's and 1970's arose from the growing importance of nuclear power generation. Caused by the strongly exothermic fission process the nuclear fuel pellets are subject to strong temperature gradients and degrade continuously by thermomigration of its components (e.g. see Bober and Schumacher, ${ }^{13)}$ Millot and Gerdanian $^{14)}$ and Janek and Timm ${ }^{15}$ ). With the increasing application of ceramic multicomponent materials at high temperatures the interest in degradation processes driven by external potential gradients (either in chemical, electric, temperature or mechanical stress fields; cf. Schmalzried ${ }^{16)}$ ) grew in general. However, the number of experimental studies on thermodiffusion in ceramic solid solutions remained very small, see section 3 for examples.

Parallel to the different experimental studies a number of theoretical approaches towards an understanding of thermodiffusion by defects in solids have been put forward, as 
summarized by Allnatt and Chadwick. ${ }^{1)}$ In the past few years, only limited theoretical and experimental work has been reported, see e. g. Timm and Janek on the Soret effect in $\mathrm{Cu}_{2-\delta} \mathrm{O}^{17)}$ or Yoo and Hwang on thermodiffusion in yttria-stabilized zirconia. ${ }^{18)}$ With the current interest in high temperature thermoelectrics this may change.

It is the purpose of the present paper to summarize some essential findings on thermodiffusion in non-metallic solids and to include references to related work. We avoid recalling the complete theoretical framework, and rather concentrate on the underlying physical ideas. Before going into detail, some specific aspects of diffusion in the solid state will be pointed out, in order to help those readers who are not so familiar with the solid state and with defect theory. Compared to investigations of the fluid state, diffusion in the solid (crystalline) state provides some unique features: First of all, crystalline solids can be regarded as ordered mixed phases (Wagner and Schottky $\left.{ }^{5}\right)$. In ionic crystals generally one rigid sublattice exists which provides a natural frame of reference for diffusion studies. As consequence of the high degree of order of the lattice, at non-zero temperature each crystal contains a thermodynamically defined concentration of equilibrium point defects. These point defects are mobile and are the necessary prerequisite for diffusion of crystal components. The motion of point defects occurs by thermally activated jumps which are characterized by a single migration enthalpy (activation barrier) for each individual jump configuration. Thus, diffusion in the solid state is based on individual jumps which are equal in respect to their physics ("hops"). In effect, the correlation between microscopic and macroscopic processes is straightforward (see Allnatt and Lidiard ${ }^{3)}$ for a detailed description), and in simple cases a single microscopic process is sufficient to interpret macroscopic mass fluxes. Very early this led in the case of thermodiffusion to attempts to find a direct correlation between the activation energy for the major transport-relevant microscopic jumps and the heat of transport - which is introduced in some detail in section 2 .

However, there are also specific features which often complicate diffusion studies in the solid state. In addition to equilibrium defects, each crystal contains non-equilibrium defects of different dimensionality (dislocations, grain boundaries, pores, inclusions). These extended defects provide fast-diffusion paths and may disturb the interpretation of diffusion studies on the basis of point defect motion. Less severe but as complicated is the presence of elastic strain due to the rigidity of the crystal lattice. Any local change by concentration or temperature gradients causes elastic strain which will influence the dynamics of the lattice. In our experimental studies we neglect both aspects.

The paper is organized as follows: In section 2 we briefly describe the necessary flux equations in the framework of linear irreversible thermodynamics, discuss the definition of the heat of transport and its relation to measurable quantities. In section 3 we review some major experimental studies of thermodiffusion and the Soret effect in crystalline solids. The reader who is more interested in the theory of the heat of transport is referred to the corresponding literature, see
Ref. 19 for brief survey.

\section{Flux equations, the Heat of transport and the Soret effect}

\subsection{Empirical approach}

In the empirical description of matter transport by diffusion, a temperature gradient creates an independent driving force which adds to the other major driving forces like concentration gradients and the electric field. Thus, if we consider a system with mobile component $k$, Fick's first empirical law is simply expanded by that temperature gradient term:

$$
\boldsymbol{j}_{k}=c_{k} v_{k}=-c_{k}\left(\tilde{D}_{k} \frac{\nabla c_{k}}{c_{k}}+D_{k}^{T} \frac{\nabla T}{T}\right)
$$

In eq. (1) the chemical diffusion coefficient $\tilde{D}_{k}$ relates the flux $\boldsymbol{j}_{k}$ of component $k$ to the molar concentration gradient $\nabla c_{k}$, and the thermodiffusion coefficient $D_{k}^{T}$ relates the flux of $k$ to the temperature gradient $\nabla T$. The sum of the gradient terms in the bracket can be interpreted as the velocity $\boldsymbol{v}_{k}$ of the mobile component $k$. Rewriting that term according to the relation

$$
v_{k}=b_{k} \boldsymbol{F}
$$

which defines the mobility $b_{k}$ of the mobile component subject to a force $\boldsymbol{F}$, we obtain:

$$
v_{k}=-\left[\tilde{b}_{k} R T \nabla \ln c_{k}+b_{k}^{T} \nabla(R T)\right]
$$

with $\tilde{b_{k}}=\tilde{D}_{k} / R T$ denoting the "chemical" mobility and with $b_{k}^{T}=D_{k}^{T} / R T$ denoting the thermodiffusive mobility. The empirical diffusion coefficients $\tilde{D}_{k}$ and $D_{k}^{T}$ base on the same (self) diffusion coefficient $D_{k}$. This self diffusion coefficient is directly related to the microscopic jump processes of the mobile component and describes the statistical motion in an isothermal system. In the case of chemical diffusion, the self diffusion coefficient is multiplied (or better: amplified) by the so-called thermodynamic factor $\left(\left(\nabla \ln a_{k} / \nabla \ln c_{k}\right)_{T}\right)$. In the case of thermodiffusion the self diffusion coefficient is multiplied by the so-called heat of transport (normalized by $R T), Q_{k}^{* \prime} / R T$, as shown in the following flux equation which can also be derived from linear irreversible thermodynamics, see below.

$$
\boldsymbol{j}_{k}=-c_{k} D_{k}\left[\left(\frac{\partial \ln a_{k}}{\partial \ln c_{k}}\right) \frac{\nabla c_{k}}{c_{k}}+\frac{Q_{k}^{* \prime}}{R T} \frac{\nabla T}{T}\right]
$$

Whereas the thermodynamic factor is well understood within thermodynamics of point defects, the heat of transport is yet not fully understood, see section 3 . However, we can get at least some rough idea of the mechanism of thermodiffusion by considering the physics of heat flow in a solid: If we neglect heat conduction by electrons (which otherwise would be included as so-called electron wind effect), phonons move 
along the temperature gradient and transport heat from the hot end to the cold end of a sample. Thermodiffusion of ions or atoms is caused when these phonons interact with mobile atomic defects, leading to a transfer of momentum and directed jumps along the temperature gradient. Unfortunately, it is not immediately obvious whether the phonon-defect interaction causes defect jumps down or up the temperature gradient - in particular if diffusion takes place via different atomic defect like vacancies or interstitials.

\subsection{Linear irreversible thermodynamics}

The formal analysis of transport phenomena in the framework of irreversible thermodynamics bases on the correct choice of forces and fluxes which have to describe the entropy production correctly (using vector/matrix notation): ${ }^{20)}$

$$
\dot{\sigma} \cdot T=\boldsymbol{J} \cdot \boldsymbol{X}
$$

Linear flux equations relate the thermodynamic forces to the corresponding independent fluxes in the linear approximation:

$$
\boldsymbol{J}=\hat{\boldsymbol{L}} \cdot \boldsymbol{X}
$$

Two major sets of forces and fluxes exist in the description of non-isothermal transport processes ${ }^{3)}$ (in the following we will change from vector notation to scalar notation, thus reducing our formal consideration to a simple one-dimensional case): a) Formulating the flux of (internal) energy, $j_{Q}$, the forces result as

$$
X_{Q}=-\frac{\nabla T}{T} \quad X_{k}=-T \nabla\left(\frac{\tilde{\mu}_{k}}{T}\right)
$$

with $\tilde{\mu}_{k}=\mu_{k}+z_{k} F \phi$ denoting the electrochemical potential of a charged component. b) Introducing the "reduced" heat flux, $j_{Q^{\prime}}$, by the transformation $j_{Q^{\prime}}=j_{Q^{-}}-\sum_{k}^{m} h_{k} j_{k}$ we obtain the following two forces:

$$
X_{Q^{\prime}}=-\frac{\nabla T}{T} \quad X_{k}^{\prime}=-\nabla\left(\tilde{\mu}_{k}\right)_{T}=-\nabla \tilde{\mu}_{k}-s_{k} \nabla T
$$

Since it is the reduced heat of transport which controls the Soret effect and which can be determined experimentally, we will make use of the second set in eq. (8) for the discussion of experiments. Eq. (7) and the heat flux $j_{Q}$ are of favorable use in computations, since $j_{\mathrm{Q}}$ is based on the internal energy rather on heat. The so-called heats of transport of the mobile components are introduced as combinations of different transport coefficients via the mathematical transformation

$$
L_{k Q^{\prime}}=\sum_{i=1}^{n} L_{k i} Q_{i}^{* \prime}
$$

and enter into the flux equations like

$$
\begin{aligned}
& j_{k}{ }^{\prime}=\sum_{i=1}^{n} L_{k i}\left(X_{i}^{\prime}+Q_{i}^{* \prime} X_{Q^{\prime}}\right) \\
& j_{Q^{\prime}}=\sum_{i=1}^{n} Q_{i}^{* \prime} \cdot j_{i}^{\prime}+\left(L_{Q Q^{-}} \sum_{i=1}^{n} L_{i Q} Q_{i}^{* \prime}\right) X_{Q^{\prime}}
\end{aligned}
$$

For further details of this transformation see Refs. 20 and 21 . Here it is sufficient to note, that $j_{k}{ }^{\prime} \equiv j_{k}$ and $L_{i k}{ }^{\prime} \equiv L_{i k}$, but that $L_{i Q^{\prime}}, L_{Q Q^{\prime}}$ and $Q_{i}^{* \prime}$ differ from $L_{i Q}, L_{Q Q}$ and $Q_{i}^{*}$.

The use of heats of transport rather than thermal diffusion coefficients has frequently been criticized but provides an intuitive approach to the interpretation of thermodiffusion, as will be shown below. In an isothermal system $\left(X_{Q^{\prime}}=0\right)$ eq. (11) can be simplified to an expression which gives a direct relation between the reduced heat flux $j_{Q^{\prime}}$ and the matter fluxes $j_{k}^{\prime}$ :

$$
j_{Q}=\sum_{k=1}^{n} Q_{k}{ }^{{ }^{*}} j_{k}{ }^{\prime}
$$

The reduced heat of transport $Q_{k}{ }^{{ }^{*}}$ of a mobile component $k$ describes the amount of heat in $\mathrm{J}$ which is transferred by a unit flux $j_{k}{ }^{\prime}=1 \mathrm{~mol} \mathrm{~m}^{2} \mathrm{~s}^{1}$ of the respective component. Correspondingly, the heat of transport $Q_{k}^{*}$ of a mobile component $k$ is the amount of (internal) energy which is transferred by a unit flux $j_{k}{ }^{\prime}$ of the respective component.

It may easily be that the reader is confused by the use of different heats of transport. In fact, the definition of the heat flux, and thus, of the heat of transport are a problem which frequently led to confusion in literature. De Groot distinguished five different heat fluxes and the corresponding sets of forces. ${ }^{20)}$ However, only the heat flux $j_{\mathrm{Q}}$ and the so-called reduced heat flux $j_{Q^{\prime}}$ are of relevance in the present context. And it is the reduced heat of transport which is determined experimentally and which describes the Soret effect, i. e. the demixing of a solid solution in a temperature gradient, in the simplest way. Rewriting eq. (10) by the use of the differential

$$
\nabla \mu_{i}=\left(\frac{\partial \mu_{i}}{\partial c_{i}}\right)_{T} \quad \nabla c_{i}+\left(\frac{\partial \mu_{i}}{\partial T}\right)_{c_{i}} \nabla T
$$

and eq. (8) we obtain

$$
j_{k}^{\prime}=-\sum_{i=1}^{n}\left[L_{k i}\left(\frac{\partial \mu_{i}}{\partial c_{i}}\right) \nabla c_{i}+L_{k i} z_{i} F \nabla \phi+L_{k i} Q_{i}^{* \prime} \frac{\nabla T}{T}\right]
$$

By introducing the thermal diffusion coefficient $D_{k}^{T}$, the chemical diffusion coefficient $\tilde{D}_{k}$ and the electric conductivity $\sigma_{k i}$ of the component $i$

$$
D_{k i}^{T}=\frac{L_{k i} Q_{i}^{* \prime}}{c_{i}} \quad \tilde{D}_{k i}=L_{k i}\left(\frac{\partial \mu_{i}}{\partial c_{i}}\right)_{T} \quad \sigma_{k i}=L_{k i}\left(z_{i} F\right)^{2}
$$

eq. (14) can be written as

$$
j_{k}^{\prime}=-\sum_{i=1}^{n}\left[\tilde{D}_{k i} \nabla c_{i}+\frac{\sigma_{k i}}{z_{i} F} \nabla \phi+n_{k i} D_{k i}^{T} \frac{\nabla T}{T}\right]
$$

If no concentration gradient $\nabla c_{i}$ and no electric field $\nabla \phi$ is present, the thermal diffusion coefficient $D_{k}^{T}$, respectively the reduced heats of transport $Q_{t}^{* \prime}$ determine the value and the sign of the flux of the component $k$ in a non-isothermal system. It has to be noted that the off-diagonal coefficents $L_{k i}$ with $k \neq i$ are usually neglected which simplifies the flux equations considerably (see Janek et al. ${ }^{22-24)}$ ). 
Rewriting eq. (10) only by insertion of eq. (8) one obtains

$$
j_{k}^{\prime}=\sum_{i=1}^{n} L_{k i}\left[\nabla \tilde{\mu}_{i}+\left(s_{i}+\frac{Q^{* \prime}}{T}\right) \nabla T\right]
$$

which also forms the basis of Wagner's discussion of thermoelectric cells involving mixed conductors. ${ }^{9)}$ Both eqs. (16) and (17) can directly be used for the discussion of the possible experimental arrangements. ${ }^{21)}$

Eq. (17) is the right starting point for the discussion of practical cases. A still relatively simple case is a binary nonstoichiometric compound with one mobile component, e. g. an oxide with mobile cations and electrons (or electron holes depending on the type of disorder). Thus, we have to regard the coupled diffusion of ions $\mathrm{A}^{\mathrm{z}}$ and electrons $\mathrm{e}$ in a component in a temperature gradient $\nabla T$. The isothermal experiment would be chemical diffusion of the metal $A$ in the chemical potential gradient $\nabla \mu_{\mathrm{A}}\left(\right.$ with $\mu_{\mathrm{A}}=\tilde{\mu}_{\mathrm{A}^{+}}+z \tilde{\mu}_{e^{-}}$). The fluxes of ions $j_{A^{z^{+}}}$and electrons $j_{e^{-}}$are coupled by the condition of electroneutrality (Nernst-Planck coupling):

$$
j_{A}=j_{A^{z^{+}}}+z j_{e^{-}}
$$

This leads to a further reduction of the two flux equations of ions and electrons as written according to eq. (17) to a single flux equation for the metal component $\mathrm{A}$ :

$$
j_{A}=-L_{A}\left[\nabla \mu_{A}+\left(s_{A}+\frac{Q_{A}^{* \prime}}{T}\right) \nabla T\right]
$$

with the transport coefficient $L_{\mathrm{A}}$ :

$$
L_{A}=\frac{L_{A^{+}} L_{e^{-}}}{L_{A^{+}}+L_{e}}
$$

the reduced heat of transport $Q_{A}^{* \prime}$ :

$$
Q_{A}^{* \prime}=Q_{A^{+}}^{* \prime}+Q_{e}^{* \prime}
$$

and the chemical potential gradient $\nabla \mu_{A}$ and partial molar entropy $s_{\mathrm{A}}$ :

$$
\nabla \mu_{A}=\nabla \tilde{\mu}_{A^{+}}+\nabla \tilde{\mu}_{e^{-}} \quad s_{A}=s_{A^{+}}+s_{e^{-}}
$$

In essence no new independent transport coefficients are obtained. Rather eqs. (17) and (19) contain the six independent transport coefficients which have been defined above. Eqs. (19) for the neutral component and (17) for the ions and electrons are the relevant equations for the experimental study of thermal diffusion in mixed conducting compounds. Clearly, other systems with different defect structures can be described by an analogous formalism. The final equations that contain the experimental observables, always appear like eq. 19 for neutral components or like eq. 17 for charged particles (see also Ref. 21).

\subsection{The Soret effect}

The working equation for the study of the Soret effect is easily derived from eq. (19), as follows. As a consequence of thermal diffusion, a temperature gradient leads to the establishment of a definite concentration gradient in the stationary state - provided that the system is finite and closed for matter exchange at its ends. The stationary concentration gradient $\nabla c_{\mathrm{A}}$ of a component $\mathrm{A}$ for this steady state $\left(j_{\mathrm{A}}=0\right)$ follows from eq. (19) by using the total differential for $\mu_{\mathrm{A}}$ (see eq. 13) as:

$$
\left.\nabla c_{A}\right|_{j_{A=0}}=-\frac{Q_{A}^{* \prime}}{\left(\frac{\partial \mu_{A}}{\partial c_{A}}\right)_{T}} \cdot \frac{\nabla T}{T}
$$

In materials with small concentration variations (i.e. deviations from stoichiometry), rather the stationary chemical potential gradient than the concentration gradient is accessible by suitable electrochemical experiments:

$$
\left.\nabla \mu_{A}\right|_{j_{A=0}}=-\left(s_{A}+\frac{Q_{A}^{* \prime}}{T}\right) \nabla T
$$

An always present difficulty in measurements of the Soret effect is the strict realization of closed surfaces $\left(j_{i}=0\right)$, and most certainly, all reported experiments on oxides suffer more or less seriously from this drawback. With respect to the evaluation of the heat of transport, the application of eq. (23) requires precise data for the thermodynamic factor, whereas the use of eq. (24) requires the knowledge of the partial entropy $s_{\mathrm{A}}$. Thus, in any case the thermodynamics of the system under investigation has to be known for the evaluation of $Q_{A}^{* \prime}$.

\section{Thermodiffusion and the Soret effect - Materials degradation in a temperature gradient}

The main problem in the analysis of thermodiffusion in solid materials is the relation of measurable macroscopic observables and the heats of transport of mobile point defects which would allow the comparison with theory. This relation is discussed in detail in Ref. 25. It is in principle not possible to measure the heat of transport of a charged point defect alone, as also other thermodynamic quantities of charged particles cannot be measured. In ionic systems one necessarily determines either the heat of transport of neutral combinations of point defects (chemical components) or the thermopower (entropy of transport) as a sum of the partial entropy $s_{k}$ and $Q_{k}{ }^{*} / T$. Of course, the same problem occurs in the liquid state: In liquid electrolytes, also only the heat of transport of neutral combinations of ions (mostly identified as the electrolyte itself) can be determined experimentally $\left(\operatorname{Haase}^{26)}\right)$. Solutions and mixtures of electrically uncharged species do not suffer from this problem.

In ionic crystals these neutral combinations are often the equivalent of neutral atoms, being composed of an ion and the dominating electronic charge carrier. Thus, in order to evaluate the heat of transport of an ion one has to introduce suitable estimates of the electronic heat of transport. Whereas reasonable estimates can be made for delocalized charge carriers 
with high mobility, ${ }^{27)}$ corresponding estimates for polarons are more difficult.

In the solid state, the Soret effect has primarily been studied in metal systems in the 1950's and 1960's, often being related to simultaneous studies of electromigration. A major review has been reported by Wever. ${ }^{10)}$ A more recent brief account with tables of data has been given by Bocquet et al. ${ }^{11)} \mathrm{A}$ more recent review on atomic transport in metals in general by Faupel ${ }^{12)}$ and includes a brief section on thermodiffusion. As stated in section 3.4, Faupel concludes that (a) the electron wind gives a major contribution to the total heat of transport and (b) essentially determines its concentration dependence at low concentrations. After substraction of this contribution a concentration independent heat of transport results. However, the results on this phonon contribution so far disclose no general relation with the migration enthalpy.

Binary nonstoichiometric compounds: If we restrict our analysis at this point to a binary mixed conductor we can identify six independent transport phenomena, in line with the formulation of flux equations (10) and (11). Three of them can be denoted as primary phenomena (ionic conduction, electronic conduction, heat conduction), since they relate principally to the diagonal transport coefficients $\left(L_{i k}\right.$ with $i=k$ ). The remaining three phenomena may be denoted as secondary or cross effects, since they relate principally to the off-diagonal transport coefficients $\left(L_{i k}\right.$ with $\left.i \neq k\right)$. One of these describes the isothermal kinetic interaction between ionic and electronic fluxes and is neglected in our present discussion. The interested reader is referred to Yoo et al. ${ }^{23)}$ and Janek. ${ }^{24)}$ The two remaining secondary phenomena can be described by the two independent heats of transport of ions and electrons (instead of using the transport coefficents $L_{i Q}$ ) and are related to a number of different transport experiments ${ }^{22,25)}$ - not all of them being independent. In essence, one can perform measurements on either electronic or ionic thermopower, on the Soret effect of the neutral mobile component, on the electronic and ionic Peltier effect and on thermodiffusion in an open system. In the present paper we will concentrate only on those experiments which offer direct information on the Soret effect.

The study of thermal diffusion in binary nonstoichiometric compounds, which are mostly mixed conductors with only one highly mobile ion (often moving via different microscopic defects), shows some major advantages compared to metal systems. Firstly, the concentration of electronic charge carriers is considerably smaller, and thus, the so-called "electron wind" plays no role in the interpretation of experimental results. Secondly, the concentration of mobile point defects can be controlled very effectively either by "self-doping" (deviation from stoichiometry) with one of the crystal components or by doping with suitable impurities.

All Soret effect experiments in these systems were performed in solid state electrochemical cells. ${ }^{28)}$ With suitable electrodes, the chemical potential gradient in the stationary case was determined, and by additional entropy measurements, the reduced heat of transport was usually evaluated. Studies of the Soret effect in mixed conductors by means of chemical potential probes (galvanic cells) have been reported for $\mathrm{CeO}_{2}$. ${ }_{\delta}{ }^{29,30)} \alpha-\mathrm{Ag}_{2+\delta} \mathrm{S},{ }^{31-34)} \beta-\mathrm{Ag}_{2+\delta} \mathrm{S},{ }^{27)} \alpha-\mathrm{Ag}_{2+\delta} \mathrm{Se}^{35,36)}$ and $\beta-\mathrm{Ag}_{2+\delta} \mathrm{Se}^{37)}$ (with $\beta$ denoting the low temperature phases) and a-AgI. ${ }^{38)}$

$\beta-\mathrm{Ag}_{2+\delta}$ Se is a true model case, as it has a very simple defect structure: The material has a narrow band gap, and thus, a high concentration of electronic defects which is virtually independent of the small metal excess. The sulfur sublattice can be considered as perfectly ordered and rigid. The cations show Frenkel disorder, and silver ions can migrate as interstitials in the interstitial sublattice and via vacancies in the regular cation sublattice. The small silver excess is large enough to influence the ionic defect concentrations, and thus, the heat of transport shows a significant concentration dependence, see Fig. 1. From the practical point of view, the results in Fig. 2 are interesting: The relative Soret effect, which describes the gradient of the metal excess driven by the temperature gradient, normalized by the metal excess, is of the order of $1 \% / \mathrm{K}$. Thus, even a small temperature difference of a small $\mathrm{K}$ leads to significant gradients of the metal excess. This gradient is negligible if we look for the absolute local metal content, but it is large if we consider defect concentrations - which control a number of important defect-related properties.

The results for another model-type phase $\left(\beta-\mathrm{Ag}_{2+\delta} \mathrm{S}\right)$ look almost the same, but the defect structure is quite different, see Figs. 3 and 4: The maximum metal excess is much smaller, and the concentration of Frenkel defects is virtually constant. The material has a wide band gap, and thus, the very low concentration of electronic defects can be changed by the

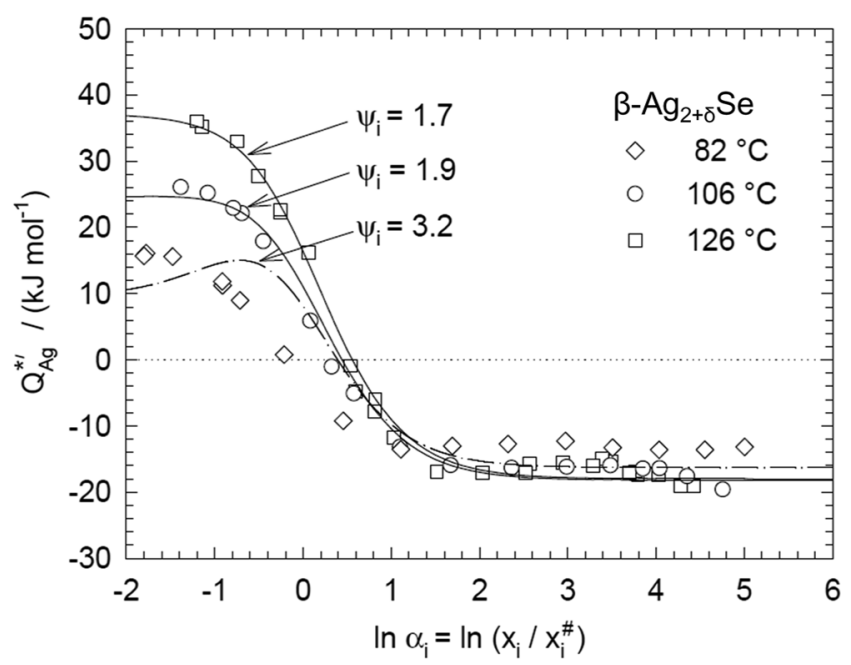

Fig. 1. Experimental results for the reduced heat of transport of $\mathrm{Ag}$ in $\beta-\mathrm{Ag}_{2+\delta} \mathrm{Se}$ (low temperature phase) as a function of the defect concentration. The phase shows cation Frenkel disorder, and the dimensionless variable $\alpha_{i}$ is a measure of the Ag excess. It describes the ratio of the actual interstitial concentration normalized by the intrinsic interstitial concentration at the stoichiometric point $\left(\alpha_{i}=0\right)$. The heat of transport shows a maximum which reflects the Frenkel defect equilibrium. ${ }^{37)} \psi_{i}$ denotes the mobility of ratio of cation interstitials and vacancies. 


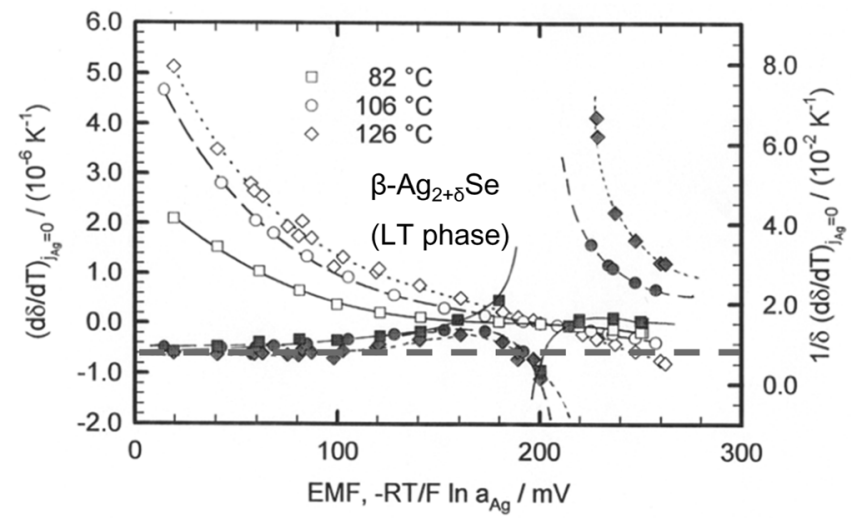

Fig. 2. Experimental results for the Soret effect of $\mathrm{Ag}$ in $\beta$ $\mathrm{Ag}_{2+\delta} \mathrm{Se}$ (low temperature phase) as a function of the silver activity in terms of the emf of a galvanic silver concentration cell. The open symbols represent the absolute Soret effect $(\mathrm{d} \delta / \mathrm{dT})$ in terms of the silver excess $\delta$ (left axis). The filled symbols represent the relative Soret effect $(\mathrm{d} \delta / \mathrm{dT}) / \delta$ which necessarily shows a singularity at the stoichiometric point. ${ }^{28)}$

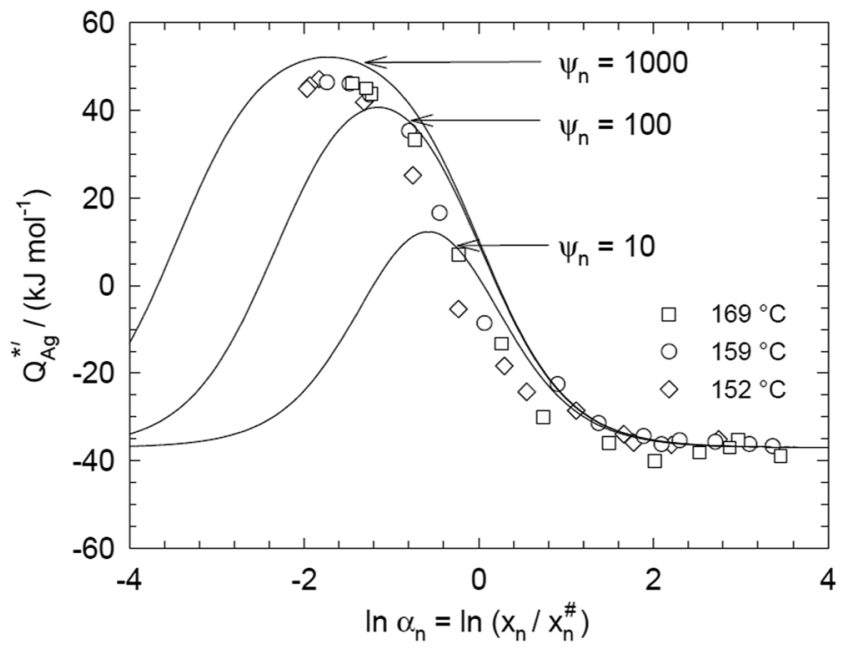

Fig. 3. Experimental results for the reduced heat of transport of $\mathrm{Ag}$ in $\beta-\mathrm{Ag}_{2+\delta} \mathrm{S}$ (low temperature phase) as a function of the defect concentration. The phase shows a composition dependent electronic disorder, and the dimensionless variable $\alpha_{n}$ is a measure of the Ag excess. It describes the ratio of the actual electron concentration normalized by the intrinsic electron concentration at the stoichiometric point $\left(\alpha_{n}=0\right)$. The heat of transport shows a maximum which reflects the band/band equilibrium. $\psi_{n}$ denotes the mobility ratio of electrons and electron holes.

small metal excess. Again, the heat of transport shows a significant concentration dependence, being due to the electronic disorder. The relative Soret effect is of the order of $2.5 \% / \mathrm{K}$ and gives rise to even stronger thermodiffusion effects on the metal excess.

The data for the high temperature phase with structural disorder in the cation sublattices look different, and the

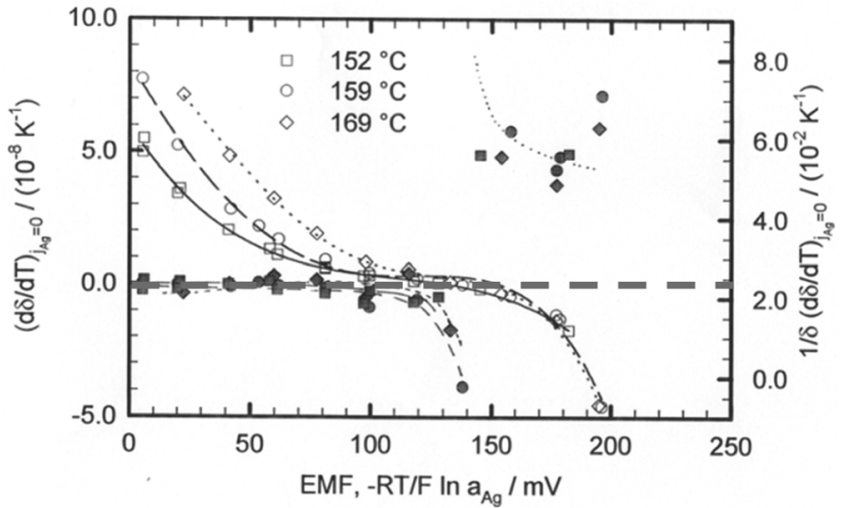

Fig. 4. Experimental results for the Soret effect of $\mathrm{Ag}$ in $\beta-\mathrm{Ag}_{2+\delta} \mathrm{S}$ (low temperature phase) as a function of the silver activity in terms of the emf of a galvanic silver concentration cell. The open symbols represent the absolute Soret effect (d $\delta / \mathrm{dT}$ ) in terms of the silver excess $\delta$ (left axis). The filled symbols represent the relative Soret effect $(\mathrm{d} \delta / \mathrm{dT}) / \delta$ which necessarily shows a singularity at the stoichiometric point. $^{28)}$

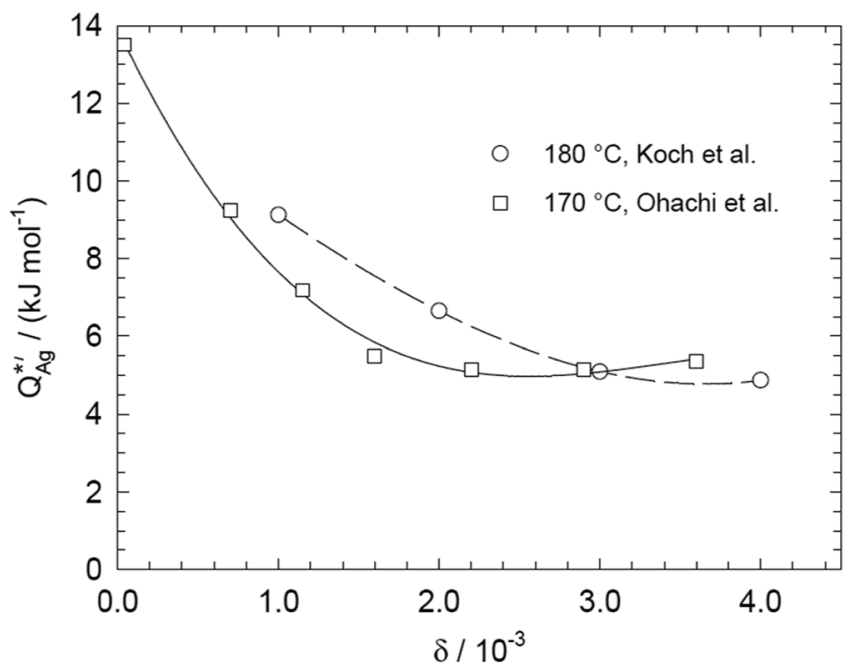

Fig. 5. Experimental results for the reduced heat of transport of silver metal in $\alpha-\mathrm{Ag}_{2+\delta} \mathrm{Se}$ as a function of the metal excess. ${ }^{35,36)}$ The data suggest that a maximum of the heat of transport exists close to the stoichiometric point.

heats of transport are smaller, see Figs. 5 and 6 . They show a strong dependence on temperature, and at least in the case of $\alpha-\mathrm{Ag}_{2+\delta}$ Se also a strong composition dependence is observed.

The only binary oxide which to best of our knowledge has been investigated so far is $\mathrm{Cu}_{2-\delta} \mathrm{O}$ (cuprite), ${ }^{17)}$ see Fig. 7. This phase shows copper deficiency which leads to cation vacancies and electron holes as majority point defects. By the use of high temperature galvanic cells Timm and Janek determined the heat of transport of $\mathrm{Cu}$ in $\mathrm{Cu}_{2-\delta} \mathrm{O}$ to be approx. $180 \mathrm{~kJ} / \mathrm{mol}$. This led to a relative Soret effect of $+1.7 \% / \mathrm{K}$ at the experimental conditions. On the basis of suitable assumptions for the 


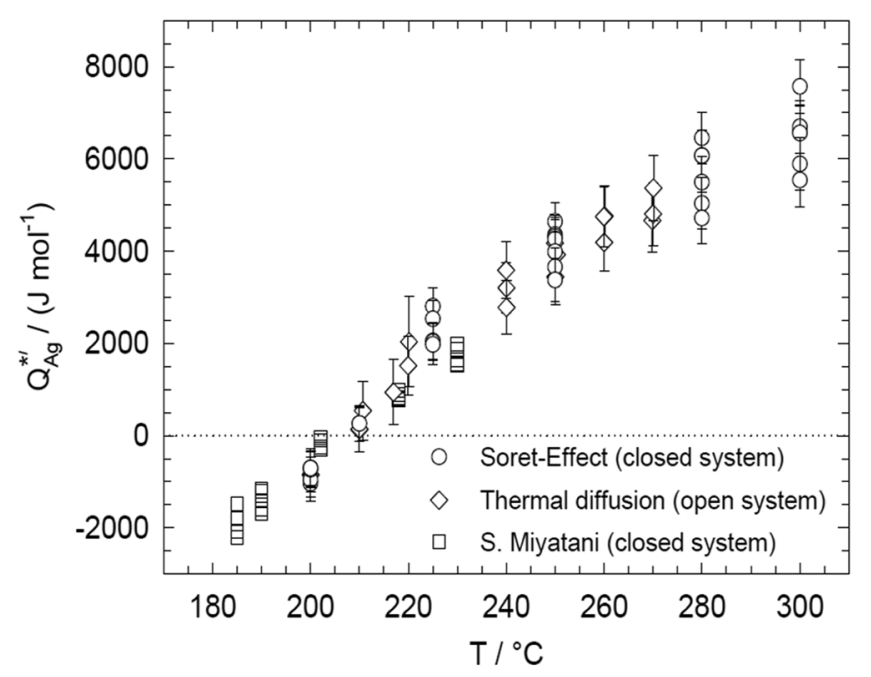

Fig. 6. Experimental results for the reduced heat of transport of silver metal in $\alpha-\mathrm{Ag}_{2+\delta} \mathrm{S}$ as a function of temperature. ${ }^{31-34)}$ The data show a pronounced temperature dependence which corresponds roughly to a slope of 8 $\mathrm{R}$ to $10 \mathrm{R}$. (a)

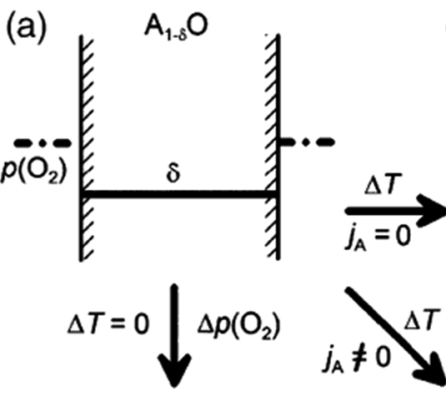

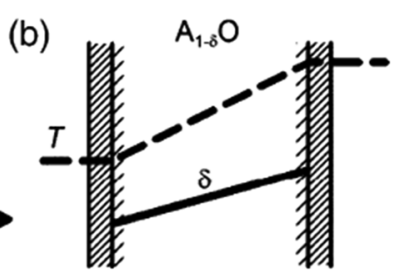
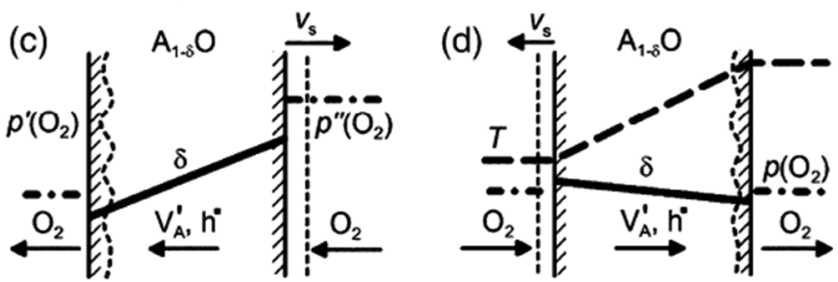

Fig. 7. Soret effect in a nonstoichiometric oxide (e. g. $\left.\mathrm{Cu}_{2 \delta} \mathrm{O}\right)$ exposed to a temperature gradient. (a) equilibrium state at given $T$ and $p\left(\mathrm{O}_{2}\right)$ showing a homogeneous metal excess, (b) Soret effect with stationary composition gradient under the influence of a stationary temperature gradient (sample with surfaces closed for matter exchange), (c) chemical diffusion and moving surfaces under the influence of a chemical potential gradient at constant temperature, (d) thermodiffusion and moving surfaces under the influence of a stationary temperature gradient with surfaces open for matter exchange.

electronic heat of transport and for the defect structure the heat of transport of $\mathrm{Cu}$ can be interpreted as the negative value of the heat of transport of the cation vacancy, $Q_{V}^{* \prime} \approx$ $180 \mathrm{~kJ} / \mathrm{mol}$. The migration enthalpy of copper vacancies equals $\Delta h_{m i g} \cong 90 \mathrm{~kJ} / \mathrm{mol}$. The result for this binary oxide with a simple defect structure shows that the assumption of equality between activation energy and heat of transport is by no means justified. In fact, Grout, Lidiard and coworkers calculate a heat of transport for vacancies in noble gas crystals which is negative and roughly twice as large as the activation energy of the vacancy jumps. ${ }^{39-43)}$

On the basis of the few available experimental data we can conclude that thermodiffusion may play an important role for the distribution of defects in a non-isothermal sample. The time to approach the stationary state is controlled by the chemical diffusion coefficient. And thus, depends sensibly on the average temperature of the sample.

\subsection{Ternary solid solutions}

As in the case of binary systems only a few case studies have been reported. Probably the best studied system is the solid solution $(\mathrm{U}, \mathrm{Pu}) \mathrm{O}_{2}$ which plays a major role as a model system for fossile fuels. (U,Pu) $\mathrm{O}_{2}$ can exist both in a hypoand a hyperstoichiometric state with respect to the oxygen content, as $\mathrm{U}^{4+}$ can either be reduced or oxidized. A detailed analysis of thermodiffusion and the Soret effect in $(\mathrm{U}, \mathrm{Pu}) \mathrm{O}_{2}$ has been reported by Timm and Janek. ${ }^{15)}$ In principle, the thermodiffusion of oxygen as a neutral component has to be considered, and the same formal description as in the case of binary nonstoichiometric compounds can be used. In fact, a pronounced minimum of the heat of transport is found close to the stoichiometric point which is another example for the strong composition dependence of the heat of transport in nonstoichiometric compounds. ${ }^{25)}$

The experimental study of the Soret effect in $(\mathrm{U}, \mathrm{Pu}) \mathrm{O}_{2}$ pellets and its analysis in terms of the heat of transport provide an interesting example for boundary conditions which do not represent 'ideal' Soret conditions: The nuclear fuel pellets are encapsulated in a metal container which provides a short-circuit for electrons, which modifies the coupling of fluxes. This is depicted schematically in Fig. 8. Details can be found in Ref. 15.

Thermodiffusion in the solid electrolyte $\mathrm{ZrO}_{2}\left(+\mathrm{Y}_{2} \mathrm{O}_{3}\right)$ has been studied by Yoo and Hwang, ${ }^{18)}$ showing nicely that the thermopower of a mixed conductor depends sensibly on the boundary conditions for matter exchange with the surrounding. Again, as in the case of $(\mathrm{U}, \mathrm{Pu}) \mathrm{O}_{2}$ the system can well be described as a quasi-binary phase with coupled diffusion of oxygen anions and electrons. Thermodiffusion in the system $\mathrm{Ag}_{1-\mathrm{x}} \mathrm{TiS}_{2}$ has been studied as the only available example for typical insertion electrodes. ${ }^{45,46)}$

Only two experimental examples for thermodiffusion in oxide solid solutions have been reported so far. Petuskey and Bowen studied the spinel phase $\mathrm{FeAl}_{2} \mathrm{O}_{4}{ }^{47}$ and performed experiments both with open samples exposed to air and with samples enclosed in an oxygen impermeable container. Interestingly, cation demixing was only observed in the case of open surfaces. In this case the temperature gradient led to a continuous flux of cation vacancies and a kinetic demixing of $\mathrm{Fe}$ and $\mathrm{Al}$. In the case of closed surfaces no measurable demixing tool place. Timm studied the solid solution $(\mathrm{Co}, \mathrm{Mg}) \mathrm{O}$ and found an appreciable demixing and also pore migration even in the case of closed surfaces. ${ }^{48}$ 


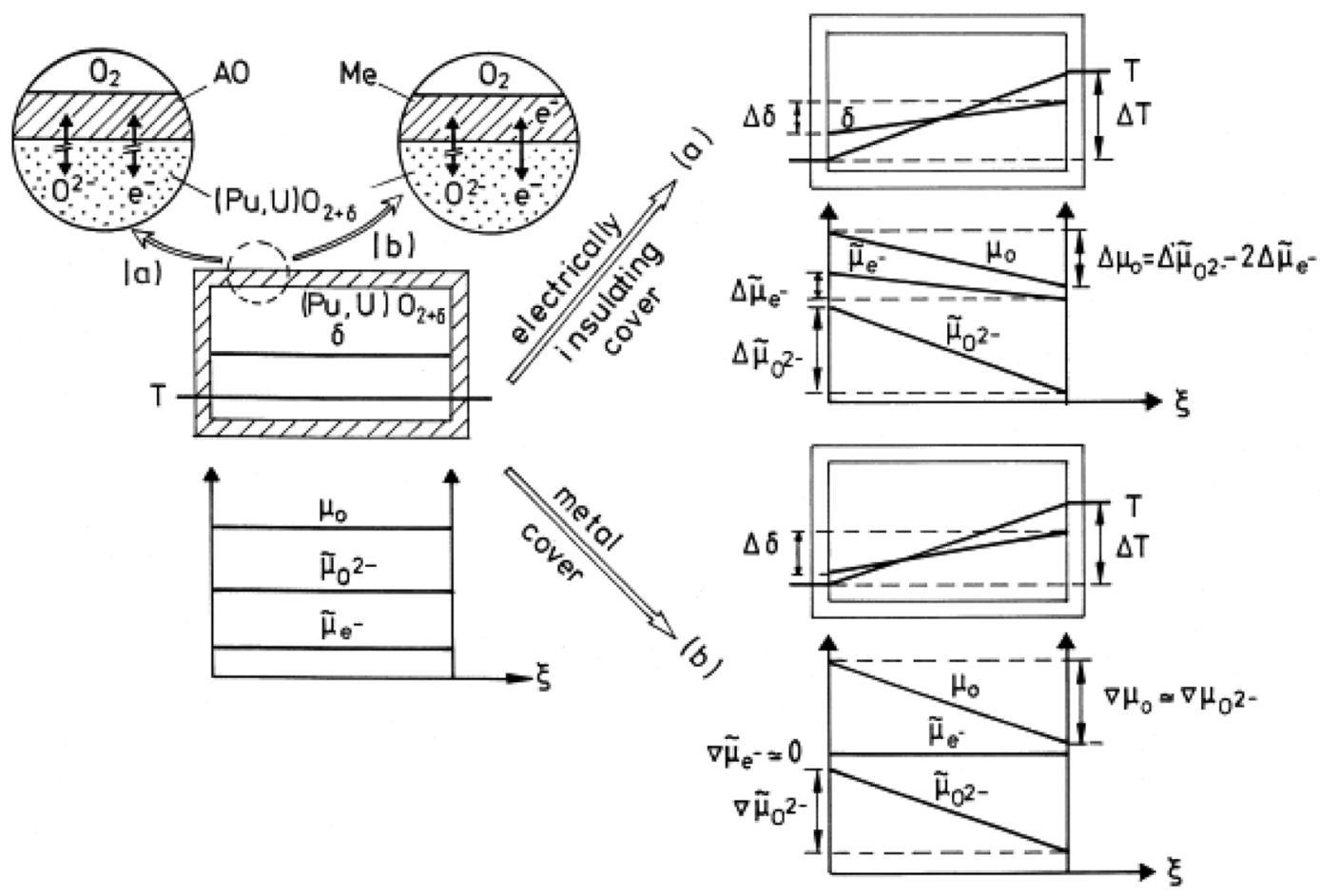

Fig. 8. Soret experiment with $(\mathrm{Pu}, \mathrm{U}) \mathrm{O}_{2}$. (a) Soret experiments with closed surfaces and inert cover, (b) Soret effect in an electronically conducting cover. Details can be found in Ref. 15.

\section{Conclusions}

Thermodiffusion and the Soret effect may lead to appreciable degradation effects in terms of demixing of solid solutions in a temperature gradient. However, our knowledge on the magnitude and the direction of the Soret effect is restricted to a few systems. And the theoretical understanding is still not very well developed. Nevertheless, a few conclusions can be drawn: a) The results for nonstoichiometric compounds show that thermodiffusion can easily lead to relative changes of the nonstoichiometry in the order of $1 \% / \mathrm{K}$. The heat of transport, which is a major factor in controlling of the Soret Effect, is both composition- and temperature-dependent. b) The Soret effect may lead to significant changes of the thermopower of nonstoichiometric compounds with time. These changes depend on the chemical boundary conditions for the non-isothermal sample, and we have to distinguish carefully between samples which are open or closed for matter exchange with the surrounding. c) Up to now there exists no simple rule of thumb for the estimation of the heat of transport for a mobile component in a solid solution. It is always the sum of the heat of transport of the mobile ion and of the heat of transport of the charge compensating electronic charge carrier. The heat of transport of electrons is usually of the order $R T$, such that the heat of transport of the ion dominates the total heat of transport. Some models predict that the heat of transport is equal to or at least of the same order as the activation energy of the mobile ion. Thus, in order to get at least a rough idea of the Soret effect for a new material, one may estimate the Soret effect on the basis of the assumption that $Q_{i}^{* \prime} \approx E_{a}$.

\section{Acknowledgments}

Financial support by the BMBF (German Ministry for Education and Research) within the project "IN-TEG" is acknowledged. The authors thank H. I. Yoo and M. Martin for continuous discussion in the field of degradation phenomena. We wish to record our appreciation of many fruitful discussions with C. Korte (Jülich), P. J. Grout (Oxford) and A. B. Lidiard (Reading) on thermodiffusion in general.

\section{REFERENCES}

1. A.R. Allnatt and A.V. Chadwick, "Thermal Diffusion in Crystalline Solids," Chem. Rev., 67 681-705 (1967).

2. W. Jost, Diffusion in Solids, Liquids and Gases, Academic Press, New York, 1964

3. A.R. Allnatt and A.B. Lidiard, Atomic Transport in Solids, Cambridge University Press, Cambridge, 1993

4. H. Reinhold, "The Thermolysis of Solid Salts (Ludwig-Soret Phenomenon)," Z. Phys. Chem. A, 141 137-40 (1929).

5. C. Wagner and W. Schottky, "Theorie der Geordneten Mischphasen (in German)," Z. Phys. Chem. B, 11 163-210 (1930).

6. R.E. Howard and A.B. Lidiard, "Thermoelectric Power of Ionic Conducting Crystals," Phil. Mag., 2 1462-67 (1957).

7. R.W. Christy, "Thermoelectric Power of Silver Halides," J. Chem. Phys., 34 1148-55 (1961).

8. E. Haga, "Theory of Thermoelectric Power of Ionic Crystals I," 
J. Phys. Soc. Jap., 13 1090-95 (1958); "Theory of Thermoelectric Power of Ionic Crystals II,” J. Phys. Soc. Jap., 14 992-96 (1959); "Theory of Thermoelectric Power of Ionic Crystals IV," J. Phys. Soc. Jap., 15 1949-54 (1960).

9. C. Wagner, "The Thermoelectric Power of Cells with Ionic Compounds Involving Ionic and Electronic Conduction," Prog. Solid State Chem., 7 1-37 (1972).

10. H. Wever, Elektro- und Thermotransport in Metallen, Julius Ambrosius Barth, Leipzig, 1973.

11. J.-L. Bocquet, G. Bŕibec, and Y. Limoge, in: Physical Metallurgy 3rd ed., vol. 1, p. 385, ed. by R.W. Cahn, P. Haasen, North-Holland, Amsterdam, 1983.

12. F. Faupel, "Atomic Transport - A Review with Perspectives," Phil. Mag. A, 65 1287-308 (1992).

13. M. Bober and G. Schumacher, "Material Transport in the Temperature Gradient of Fast Reactor Fuels," Adv. Nucl. Sci. Technol., 7 121-79 (1973).

14. F. Millot, P. Gerdanian, "Measurement of Thermo- and Electrotransport in Fluorite Oxides," J. Nucl. Mater., 92 257-61 (1980).

15. J. Janek and H. Timm, "Thermal Diffusion and Soret Effect in $\left(\mathrm{U}_{5} \mathrm{Me}\right)_{2+\delta}$ : The Heat of Transport of Oxygen," $J$. Nucl. Mater., 255 116-127 (1998)

16. H. Schmalzried: Chemical Kinetics of Solids, VCH, Weinheim, 1995.

17. H. Timm and J. Janek, "On the Soret Effect in Binary Nonstoichiometric Oxides - Kinetic Demixing of Cuprite in a Temperature Gradient," Solid State Ionics, 176 1131-43 (2005).

18. H.-I. Yoo and J.H. Hwang, "Thermoelectric Behavior of Single Crystalline $\mathrm{ZrO}_{2}\left(+8 \mathrm{~m} / \mathrm{o} \mathrm{Y}_{2} \mathrm{O}_{3}\right)$," J. Phys. Chem. Solids, 53 973-81 (1992).

19. J. Janek, C. Korte and A. B. Lidiard, in: Thermal Nonequilibrium Phenomena in Fluid Mixtures, pp. 146-183, ed. by W. Köhler and S. Wiegand, series: Lecture Notes in Physics, Springer-Verlag, Berlin, 2002.

20. S. R. de Groot, Thermodynamik irreversibler Prozesse, vol. 18/18a, Bibliographisches Institut, Mannheim, 1960.

21. C. Korte, "Nichtisotherme Transportprozesse in Gemischtleitenden Ionenkristallen," $\mathrm{Ph}$. D. Thesis, University of Hannover, 1997.

22. C. Wagner, "Equations for Transport in Solid Oxides and Sulfides of Transition Metals," Prog. Solid State Chem. (Part 1), 10 3-16 (1975).

23. H.I. Yoo, H. Schmalzried, M. Martin, and J. Janek, "Cross Effect between Electronic and Ionic Flows in Semiconducting Transition Metal Oxides," Z. Phys. Chem. N. F., 168 129-42 (1990).

24. J. Janek, "Coupling between Ionic and Electronic Fluxes in Mixed Ionic/Electronic Conductors: Experiments on $\mathrm{Co}_{1-\delta} \mathrm{O}$," Ber. Bunsenges. Phys. Chem., 98 1213-23 (1994).

25. J. Janek and C. Korte, "Cross Effect between Heat and Matter Fluxes in Mixed Conducting Solids - Definition of the Heats of Transport," Z. Phys. Chem., 196 187-208 (1996).

26. R. Haase, "Thermodynamik der Irreversiblen Prozesse". In: Fortschritte der Physikalischen Chemie, Vol. 8, ed. by W. Jost, Verlag, Darmstadt, 1963

27. C. Korte and J. Janek, "Nonisothermal Transport Properties of $\alpha-\mathrm{Ag}_{2+\delta} \mathrm{S}$ : Partial Thermopowers of Electrons and Ions, the Soret Effect and Heats of Transport," J. Phys. Chem. Solids, 58 623-37 (1997).

28. C. Korte, J. Janek, and H. Timm, "Transport Processes in Temperature Gradients: Thermal Diffusion and Soret Effect in Crystalline Solids," Solid State Ionics, 101-103 465-70 (1997).

29. F. Millot and P. Gerdanian, "A New Method for the Study of Thermomigration Using a Nonisothermal Electrolytic Cell," J. Phys. Chem. Solids, 43 [6] 501-6 (1982).

30. F. Millot and P. Gerdanian, "Thermomigration dans $\mathrm{CeO}_{2-\mathrm{x}}$," J. Nucl. Mater., 116 55-62 (1983).

31. S. Miyatani, " $\alpha-\mathrm{Ag}_{2} \mathrm{~S}$ as a Mixed Conductor," J. Phys. Soc. Jap., 24 328-35 (1968).

32. J. Janek, "Thermal Diffusion in Crystalline Binary Compounds with Narrow Range of Homogeneity: I. A New Experiment for the Determination of the Heat of Transport," Ber. Bunsenges. Phys. Chem., 99 920-31 (1995).

33. J. Janek and C. Korte, "Thermal Diffusion in Crystalline Binary Compounds with Narrow Range of Homogeneity: II. Stationary Thermal Diffusion in $\alpha-\mathrm{Ag}_{2+\delta} \mathrm{S}$," Ber. Bunsenges. Phys. Chem., 99 932-39 (1995).

34. C. Korte and J. Janek, "Thermal Diffusion in Crystalline Binary Compounds with Narrow Range of Homogeneity: III. Experimental Study of the Soret Effect in $\alpha-\mathrm{Ag}_{2+\delta} \mathrm{S}$," Ber. Bunsenges. Phys. Chem., 100 425-32 (1996).

35. W. Koch, H. Rickert, and G. Schlechtriemen, "Non-isothermal Stationary States, Thermoelectric Powers and Transport Processes in $\alpha-\mathrm{Ag}_{2+\delta}$ Se in a Temperature Gradient," Solid State Ionics, 9/10 1197-204 (1983).

36. T. Ohachi and I. Taniguchi, "Measurement of the Thermal Diffusion Coefficient of $\mathrm{Ag}$ Atoms in $\alpha-\mathrm{Ag}_{2+\delta} \mathrm{Se}$," Solid State Ionics, 3/4 89-92 (1981).

37. C. Korte and J. Janek, "Ionic Conductivity, Partial Thermopowers, Heats of Transport and the Soret Effect of $\alpha$ $\mathrm{Ag}_{2+\delta} \mathrm{Se}$ - An Experimental Study," Z. Phys. Chem., 206 129-63 (1998).

38. D.-L. Kim and H.-I. Yoo, "Thermopower of an Asymmetric Cell, $\mathrm{Ag}|\mathrm{AgI}| \mathrm{Ag}_{2} \mathrm{~S}$, Involving a Mixed Conductor $\mathrm{Ag}_{2} \mathrm{~S}$ as an Information Transmitter," Solid State Ionics, 81 135-43 (1995).

39. C. Jones, P.J. Grout, and A.B. Lidiard, "The Heat of Transport of Vacancies in Solid Argon," Phil. Mag. Lett., 74 217-23 (1996).

40. C. Jones, P.J. Grout, and A.B. Lidiard, "The Heat of Transport of Solute Atoms in Solid Argon," Ber. Bunsenges. Phys. Chem., 101 1232-37 (1997).

41. C. Jones, P.J. Grout, and A.B. Lidiard, "The Heat of Transport of Vacancies in Model FCC Solids," Phil. Mag, A, 79 2051-70 (1999).

42. P. J. Grout and A. B. Lidiard, "Computation of Heats of Transport in Crystalline Solids II," J. Phys. Cond. Matter, 20425201 (2008).

43. K. A. M. Dickens, P. J. Grout, and A. B. Lidiard, "Computation of Heats of Transport of Vacancies in Model Crystalline Solids III," J. Phys. Cond. Matter, 23265401 (2011).

44. C. Sari and G. Schumacher, "Oxygen Redistribution in Fast Reactor Oxide Fuel," J. Nucl. Mater., 61 192-202 (1976).

45. A. Honders, E.W.H. Young, J.H.W. de Witt, and G.H.J. Broers, "The Thermoelectric Power in $\mathrm{Ag}_{\mathrm{x}} \mathrm{TiS}_{2}$. Part I. The Electronic Component of the Thermoelectric Power," Solid State Ionics 8 
115-19 (1983).

46. A. Honders, A.J.H. Hintzen, J.M. der Kinderen, J.H.W. de Witt, and G.H.J. Broers, "The Thermoelectric Power in Solid Solution Electrodes: A Disregarded Phenomenon?," Solid State Ionics, 9/10 1205-11 (1983).

47. W.T. Petuskey and H.K. Bowen, "Thermal Segregation of Cations in Iron-aluminate Spinels," J. Am. Cer. Soc., 64
611-17 (1981).

48. H. Timm, Thermodiffusion in binären und ternären Oxiden. Ph. D. Thesis, University of Hannover, Germany (1999). 49. J. Janek and C. Korte, "Study of the Soret Effect in Mixed Conductors by the Measurement of Ionic and Electronic Thermopower," Solid State Ionics, 92 193-204 (1996). 